Article

\title{
Long Term Real-World Outcomes of Trifluridine/Tipiracil in Metastatic Colorectal Cancer-A Single UK Centre Experience
}

\author{
Daniel Tong ${ }^{1}$, Lei Wang ${ }^{1}{ }^{1}$, Jeewaka Mendis ${ }^{2}$ and Sharadah Essapen ${ }^{1, *}$ \\ 1 St Luke's Cancer Centre, Royal Surrey NHS Foundation Trust, Egerton Road, Guildford GU2 7XX, UK; \\ dtong@nhs.net (D.T.); l.wang1@nhs.net (L.W.) \\ 2 Surrey Clinical Trials Unit, University of Surrey, Egerton Road, Guildford GU2 7XP, UK; \\ a.mendis@surrey.ac.uk \\ * Correspondence: s.essapen@nhs.net
}

check for

updates

Citation: Tong, D.; Wang, L.; Mendis, J.; Essapen, S. Long Term Real-World Outcomes of Trifluridine/Tipiracil in Metastatic Colorectal Cancer-A Single UK Centre Experience. Curr. Oncol. 2021, 28, 2260-2269. https:// doi.org/10.3390/curroncol28030208

Received: 27 March 2021

Accepted: 15 June 2021

Published: 18 June 2021

Publisher's Note: MDPI stays neutral with regard to jurisdictional claims in published maps and institutional affiliations.

Copyright: (c) 2021 by the authors. Licensee MDPI, Basel, Switzerland. This article is an open access article distributed under the terms and conditions of the Creative Commons Attribution (CC BY) license (https:// creativecommons.org/licenses/by/ $4.0 /)$.

\begin{abstract}
In the UK, Trifluridine-tipiracil (Lonsurf) is used to treat metastatic colorectal cancer in the third-line setting, after prior exposure to fluoropyrimidine-based regimes. Current data on the real-world use of Lonsurf lack long-term follow-up data. A retrospective evaluation of patients receiving Lonsurf at our Cancer Centre in 2016-2017 was performed, all with a minimum of two-year follow-up. Fifty-six patients were included in the review. The median number of cycles of Lonsurf administered was 3. Median follow-up was 6.0 months, with all patients deceased at the time of analysis. Median progression-free survival (PFS) was 3.2 months, and overall survival (OS) was 5.8 months. The median interval from Lonsurf discontinuation to death was two months, but seven patients received further systemic treatment and median OS gained was 12 months. Lonsurf offered a slightly better PFS but inferior OS to that of the RECOURSE trial, with PFS similar to real-world data previously presented. Interestingly, $12.5 \%$ had a PFS $>9$ months, and this cohort had primarily left-sided and RAS wild-type disease. A subset received further systemic treatment on Lonsurf discontinuation with good additional OS benefit. Lonsurf may alter the course of disease for a subset of patients, and further treatment on progression can be considered in carefully selected patients.
\end{abstract}

Keywords: Trifluridine-tipiracil; Lonsurf; TAS-102; real-world; colorectal cancer

\section{Introduction}

In the UK, colorectal cancer (CRC) is the fourth most common cancer, and the second commonest cause of cancer deaths [1]. First-line palliative chemotherapy often consists of a fluoropyrimidine (Capecitabine or 5-Fluorouracil), and in patients who are fit and of good performance status, this is usually combined with oxaliplatin or irinotecan. Furthermore, where the tumour does not carry an RAS mutation, the addition of an anti-epidermal growth factor receptor inhibitor (e.g., Cetuximab or Panitumumab) to one of these combinations is recommended to achieve the longest overall survival (OS) [2]. The addition of anti-VEGF therapy or anti-EGFR therapy in the absence of RAS mutations can further improve the benefit from chemotherapy [3].

Trifluridine-tipiracil (Lonsurf) is an oral combination of the antineoplastic nucleoside analogue trifluridine (FTD) and the thymidine phosphorylator inhibitor tipiracil (TPI) [4]. Tipiracil has both a potentiating effect on trifluridine, and a separate anti-angiogenic effect. A phase II study from Japan and the subsequent phase III RECOURSE trial demonstrated the safety and efficacy of Lonsurf in 2014 and 2015, respectively [5,6]. Lonsurf showed efficacy against placebo in the RECOURSE trial, improving overall survival from 5.3 months to 7.1 months, with a hazard ratio (HR) of 0.68 [6]. Progression-free survival (PFS) was improved with a hazard ratio of 0.48 . The response rate was only $1.6 \%$ but disease control rate, which includes stable disease, was $44 \%$, as compared to $16 \%$ with placebo. The commonest adverse event was neutropenia, and there was one treatment-related death. Based on the trial findings, Lonsurf then obtained FDA approval for the treatment of advanced 
colorectal cancer [7]. These studies led to this drug being made available in Europe and the UK in 2016, in refractory mCRC. In the UK, Lonsurf is the only agent approved by the National Institute for Health and Care Excellence (NICE) for use in patients who have become refractory to fluoropyrimidine, oxaliplatin and irinotecan chemotherapy [8].

With regard to real-world outcomes, PRECONNECT is a large multi-center international Phase IIIlb study with 798 patients [9]. It has reported safety, quality of life and PFS results. No OS data are available due to the short follow-up of just 28 days after treatment termination. Median PFS was 2.8 months, an improvement on the results of RECOURSE. Other data available include published retrospective data, meta-analysis and abstracts [10-15]. Their reported outcomes are summarised in Table 1.

Table 1. A summary of pre-existing Lonsurf real-world outcomes compared with trial data.

\begin{tabular}{|c|c|c|c|c|c|c|c|c|c|}
\hline $\begin{array}{c}\text { Study } \\
\text { [Reference] }\end{array}$ & Country & Study Type & $\begin{array}{c}\text { Number } \\
\text { of Patients }\end{array}$ & $\begin{array}{l}\text { Performance } \\
\text { Status }\end{array}$ & $\begin{array}{c}\text { RAS } \\
\text { Mutant } \\
(\%)\end{array}$ & $\begin{array}{l}\text { Previous } \\
\text { Lines of } \\
\text { Treatment }\end{array}$ & $\begin{array}{c}\text { Median } \\
\text { Duration of } \\
\text { Treatment } \\
\text { (months) }\end{array}$ & $\begin{array}{c}\text { Median } \\
\text { PFS } \\
\text { (months) }\end{array}$ & $\begin{array}{c}\text { Median } \\
\text { OS } \\
\text { (months) }\end{array}$ \\
\hline RECOURSE [6] & International & $\begin{array}{c}\text { Phase III } \\
\text { trial }\end{array}$ & 800 & $0-1$ & 51 & $\geq 2$ & 1.5 & 2 & 7.1 \\
\hline PRECONNECT [9] & International & $\begin{array}{c}\text { Phase IIIlb } \\
\text { trial }\end{array}$ & 798 & $0-1$ in $97 \%$ & 52.6 & $\begin{array}{c}36.1 \% \leq 2 \\
30.6 \% 3 \\
32.8 \% \geq 4\end{array}$ & 2.8 & 3 & $\begin{array}{c}\text { Not } \\
\text { reported }\end{array}$ \\
\hline Stavraka et al. [10] & UK & $\begin{array}{l}\text { Abstract } \\
\text { (retrospec- } \\
\text { tive) }\end{array}$ & 236 & $0-1$ in $90 \%$ & $\begin{array}{c}\text { Not } \\
\text { re- } \\
\text { ported }\end{array}$ & $\geq 2$ & 3 & 3.3 & 7.6 \\
\hline Tilby et al. [11] & UK & $\begin{array}{l}\text { Abstract } \\
\text { (retrospec- } \\
\text { tive) }\end{array}$ & 91 & $0-1$ in $96 \%$ & 53 & Not reported & 4.3 & 4.1 & 8.7 \\
\hline Samawi et al. [12] & Canada & $\begin{array}{l}\text { Retrospective } \\
\text { study }\end{array}$ & 717 & Not stated & 60 & Not reported & 2.5 & $\begin{array}{l}\text { Not } \\
\text { reported }\end{array}$ & $\begin{array}{c}\text { Not } \\
\text { reported }\end{array}$ \\
\hline Anderson et al. [13] & $\begin{array}{l}\text { Japan, } \\
\text { Europe }\end{array}$ & $\begin{array}{l}\text { Meta- } \\
\text { analysis }\end{array}$ & 1008 & $0-1$ in $93 \%$ & 62 & $\begin{array}{c}34 \% \leq 2 \\
35 \% 3 \\
31 \% \geq 4\end{array}$ & $\begin{array}{c}\text { Pooled data } \\
\text { not reported, } \\
\text { individual } \\
\text { studies ranged } \\
\quad 1-3\end{array}$ & 2.2 & 6.6 \\
\hline Wallander et al. [14] & Sweden & $\begin{array}{l}\text { Retrospective } \\
\text { study }\end{array}$ & 48 & $0-1$ in $94 \%$ & 60 & $\begin{array}{c}73 \% \leq 2 \\
23 \% 3 \\
2 \% \geq 4\end{array}$ & Not reported & 2.3 & 6.4 \\
\hline Cremolini et al. [15] & Italy & $\begin{array}{l}\text { Retrospective } \\
\text { study }\end{array}$ & 341 & $0-1$ in $98 \%$ & 59 & $\begin{array}{c}33.4 \% \leq 2 \\
28.1 \% 3 \\
38.4 \% \geq 4\end{array}$ & Not reported & 2.4 & 6.2 \\
\hline
\end{tabular}

PFS (progression-free survival), OS (overall survival).

A limitation of the pre-existing real-world data is the lack of long-term follow-up. We therefore examined the outcomes for patients treated with Lonsurf at a large tertiary Cancer Centre in the southeast of the United Kingdom, with a minimum of two years follow-up. Our study is the largest published review from a single institution in the United Kingdom.

\section{Materials and Methods}

In this retrospective review, data were collected on all metastatic colorectal cancer (mCRC) patients treated with Lonsurf at a tertiary Cancer Centre between 2016 and 2017. Patients without a minimum of two years follow-up, unless due to death, were excluded. Data were collected from electronic patient notes, analysed and stored in a secured Trust network drive. Data collected included baseline demographics and tumour characteristics, treatment details prior to, during and after Lonsurf, and treatment outcomes.

All patients had undergone KRAS and NRAS assessment of their cancer. Assays were performed using the Cobas KRAS Mutation Test Kit (Roche, London, UK) and the NRAS mutation detection kit (Entrogen, Longfield, UK). The assessment for BRAF mutation was performed by next generation sequencing on the Ion Torrent PGM platform (ThermoFisher, Paisley, UK). 


\section{Statistical Analysis}

Kaplan-Meier curves, graphical illustrations of the survival function, are produced for both overall survival and progression-free survival using the Lifetest procedure (PROC LIFETEST) in SAS ${ }^{\circledR}$ software (SAS Institute Inc., London, UK). The median survival time is also reported for both overall and progression-free survival.

To evaluate the effect of potential predictors on the survival length, Cox proportional hazard models were fitted, separately for overall survival and progression-free survival, using Phreg procedure (PROC PHREG) in SAS ${ }^{\circledR}$ Software. This model evaluates the effect of age (the dichotomy of below or above 65 years), tumour location (left or right), RAS mutation (wildtype or mutant), metastatic at diagnosis (yes or no), sites of metastasis 3 or more (yes or no), time from diagnosis until treatment with Lonsurf greater than 18 months (yes or no), single organ metastasis (yes or no), lung only metastasis (yes or no), liver only metastasis (yes or no) on the length of survival. Resulting hazard ratios along with 95\% confidence intervals and $p$-values of one level of the predictor with respect to a reference level are reported. Statistical significance is evaluated at the conventional cut off level of $5 \%$.

\section{Results}

\subsection{Patient Demographics and Tumour Characteristics}

A total of 56 patients were included in the review. The median age was 61 years (range $37-79)$ and $59 \%$ of patients were male (Table 2$)$. Further, 40 patients $(71.4 \%$ ) had a left-sided tumour, $38(67.9 \%)$ had T3 or T4 disease at diagnosis, whilst only two (3.6\%) patients had node-negative disease at diagnosis. Thirty-six $(66 \%)$ patients had metastatic disease at diagnosis, and the median time to metastasis for the remaining cohort was 11 months (range 2-108). The liver was the first site of metastatic disease in the majority of cases $(n=39,69.6 \%)$, and 21 patients had liver-only disease. Twenty-one patients $(37.5 \%)$ had lung disease, and this was the sole site of metastasis in three patients. Sixteen patients $(28.6 \%)$ had multiple sites of metastases.

Table 2. Patient and treatment demographics prior to Lonsurf.

\begin{tabular}{ccc}
\hline Variable & Value $(\boldsymbol{n})$ & $\mathbf{( \% )}$ \\
\hline & Baseline characteristics & \\
\hline Gender & 33 & 59 \\
Male & 23 & 41 \\
Female & 61 & Range $37-79$ \\
Median age at diagnosis & & \\
Location of tumour & 40 & 71.4 \\
Left-sided & 15 & 26.8 \\
Right-sided & 1 & 1.8 \\
Other & Staging at diagnosis \\
& & \\
\hline $\mathrm{T}$ & 0 & 0 \\
1 & 2 & 3.6 \\
2 & 22 & 39.3 \\
3 & 16 & 28.6 \\
$\mathrm{~N}$ & &
\end{tabular}


Table 2. Cont.

\begin{tabular}{|c|c|c|}
\hline Variable & Value $(n)$ & $(\%)$ \\
\hline 0 & 2 & 3.6 \\
\hline 1 & 13 & 23.2 \\
\hline 2 & 25 & 44.6 \\
\hline \multicolumn{3}{|l|}{$\mathrm{M}$} \\
\hline 0 & 16 & 30.8 \\
\hline 1 & 36 & 69.2 \\
\hline $\begin{array}{l}\text { Median interval until } \\
\text { metastasis (months) }\end{array}$ & 11 & Range $2-108$ \\
\hline \multicolumn{3}{|c|}{ Site of metastasis } \\
\hline Lung & 21 & 37.5 \\
\hline Liver & 39 & 69.6 \\
\hline Peritoneum & 8 & 14.2 \\
\hline Nodal & 8 & 14.2 \\
\hline Ovary & 2 & 3.6 \\
\hline \multicolumn{3}{|c|}{ Multiple sites of metastases? } \\
\hline Yes & 16 & 28.6 \\
\hline No & 40 & 71.4 \\
\hline \multicolumn{3}{|c|}{ Histology } \\
\hline $\begin{array}{c}\text { Median number of positive } \\
\text { nodes }\end{array}$ & 4 & Range 0-18 \\
\hline \multicolumn{3}{|c|}{ Grade of tumour } \\
\hline 1 & 0 & 0 \\
\hline 2 & 32 & 57.1 \\
\hline 3 & 6 & 10.7 \\
\hline Not stated or not operated & 18 & 32.1 \\
\hline \multicolumn{3}{|c|}{ Extramural vascular invasion } \\
\hline Positive & 16 & 28.6 \\
\hline Negative & 15 & 26.8 \\
\hline Not stated & 25 & 44.6 \\
\hline \multicolumn{3}{|c|}{ RAS mutation } \\
\hline Mutant & 23 & 41.4 \\
\hline Wildtype & 25 & 44.6 \\
\hline Not tested & 8 & 14.2 \\
\hline \multicolumn{3}{|c|}{ BRAF mutation } \\
\hline Mutant & 1 & 1.8 \\
\hline Wildtype & 14 & 25 \\
\hline Not tested & 41 & 73.2 \\
\hline \multicolumn{3}{|c|}{ Systemic treatment details } \\
\hline \multicolumn{3}{|l|}{ Adjuvant chemo received } \\
\hline Yes & 22 & 39.3 \\
\hline No & 34 & 60.7 \\
\hline \multicolumn{3}{|c|}{ Regimen } \\
\hline FOLFOX & 12 & 54.5 \\
\hline FOLFIRI & 1 & 1.8 \\
\hline CAPOX & 2 & 3.6 \\
\hline $5^{\prime} \mathrm{FU}$ & 1 & 1.8 \\
\hline Capecitabine & 1 & 1.8 \\
\hline Not stated & 5 & 8.9 \\
\hline
\end{tabular}


Table 2. Cont.

\begin{tabular}{ccc}
\hline Variable & Value $(\boldsymbol{n})$ & $\mathbf{( \% )}$ \\
\hline $\begin{array}{c}\text { Median number of cycles } \\
\text { received }\end{array}$ & 12 & Range 2-14 \\
\hline & Lines received prior to LONSURF & 0 \\
2 & 0 & 92.9 \\
3 & 52 & 5.4 \\
Not stated & 3 & 1.8 \\
\hline Median FOLFOX received & 1 & Range 4-31 \\
\hline Median FOLFIRI received & 12 & Range 3-43 \\
\hline FOLFOX (Fluorouracil-Oxaliplatin), FOLFIRI (Fluorouracil-Irinotecan), LONSURF (Trifluridine-tipiracil).
\end{tabular}

All patients had undergone KRAS and NRAS assessment of their cancer. A RAS mutation was found in $23(41.4 \%)$ patients, with only one BRAF mutation identified. Only $26.8 \%$ of tumours were assessed for BRAF mutation in this study as BRAF tests were not part of routine molecular profiling requests during the period when this study cohort received Lonsurf, between 2016 and 2017.

\subsection{Systemic Treatment Received Prior to Lonsurf}

Adjuvant chemotherapy was given in $39.3 \%$ of the studied population, with 12 patients (54.5\%) receiving Fluorouracil-Oxaliplatin (FOLFOX), two having Capecitabine-Oxaliplatin (CAPOX), and one receiving Fluorouracil-Irinotecan (FOLFIRI) (Table 2). The median number of cycles of adjuvant chemotherapy received was 12 (range 2-14) for FOLFOX and FOLFIRI, and two patients both had eight cycles of CAPOX.

All mCRC patients had a minimum of two lines of systemic treatment for their metastatic disease prior to receiving Lonsurf, with three patients (5.4\%) receiving three prior lines of treatment. Median cycles of FOLFOX and FOLFIRI received prior to Lonsurf were 12 (range 4-31) and 12 (3-43), respectively.

\subsection{Lonsurf Treatment Details}

The median treatment-free interval before starting Lonsurf was 1 month (range 0-23), and the median number of cycles administered was 3 (range 1-16) (Table S1). Median follow-up was 6.0 months (range 1-28), with all patients deceased at time of analysis. The median progression-free survival was 3.2 months (range 1-18), and median overall survival was 5.8 months (1-28) (Figure 1). Seven patients went on to have further lines of systemic treatment; five of whom had Capecitabine monotherapy, while two had FOLFOX. In addition, two patients were referred for consideration of Phase 1 trials.

Among the seven patients who had subsequent therapies, median OS was 12 months (range 1-17) from the point of Lonsurf discontinuation. Four (57.1\%) patients had left-sided disease, four had metastatic disease at initial diagnosis, and four had cancers which carried a RAS mutation. The median time to second progression was 7.0 months (range 3-8). Multivariate analysis revealed patients presenting with metastatic disease and aged $>65$ at diagnosis had worse outcomes with Lonsurf (Table 3). HR for PFS was 2.7 (95\% CI 1.3 to $5.9, p=0.009)$ among those who were metastatic at diagnosis. In addition, patients aged 65 or greater at the time of diagnosis had worse OS, with HR of 2.1 (95\% CI 1.1-4.2, $p=0.03$ ). 

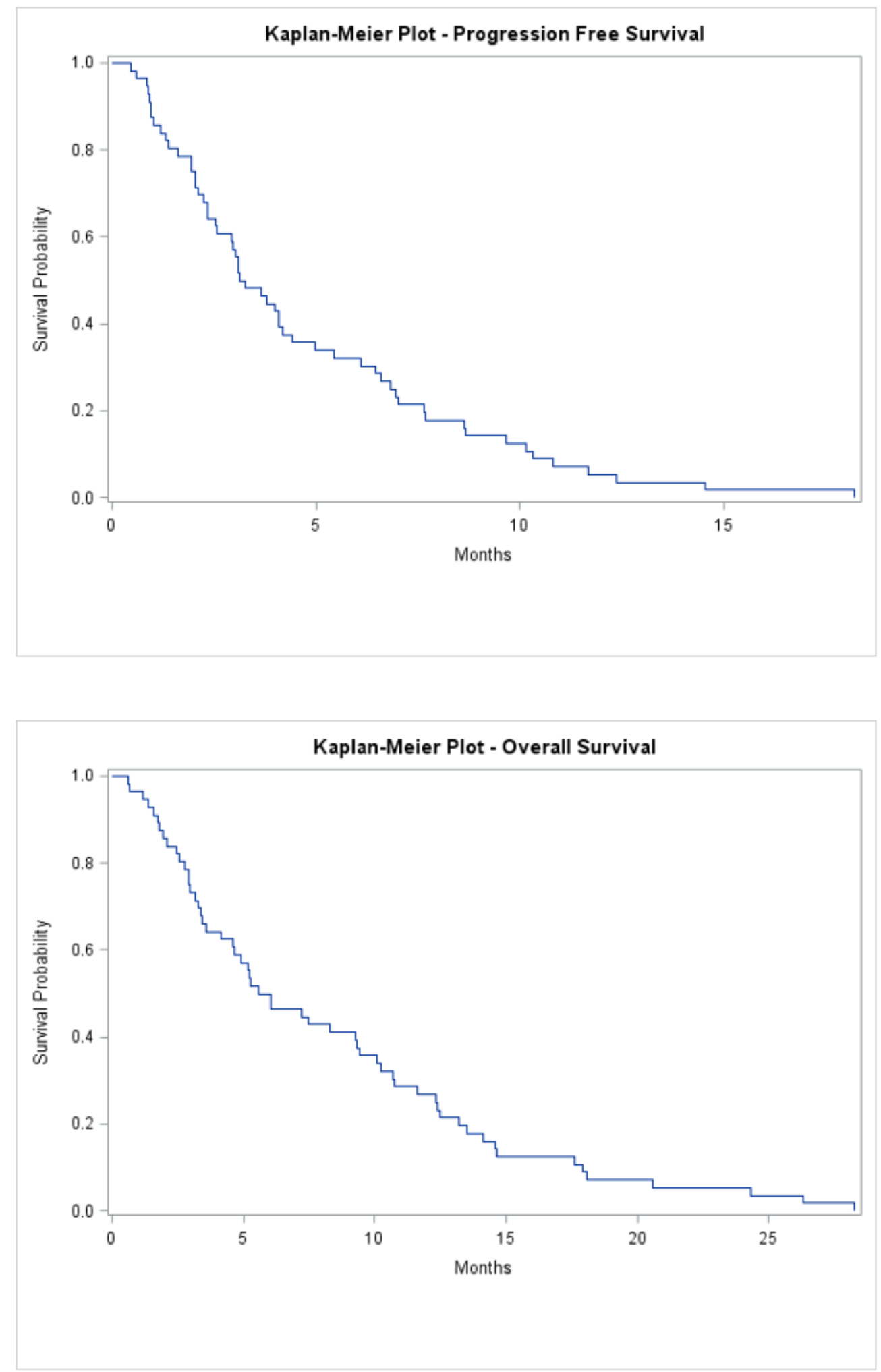

Figure 1. Kaplan-Meier progression-free survival and overall survival of all patients treated. 
Table 3. Multivariable analysis of the effect of parameters on PFS and OS.

\begin{tabular}{ccccc}
\hline Parameters & \multicolumn{2}{c}{ PFS } & \multicolumn{2}{c}{ OS } \\
\hline & HR $(95 \%$ CI $)$ & $p$-Value & HR $(95 \%$ CI $)$ & $p$-Value \\
\hline Age at diagnosis ( $\geq 65$ vs. $<65)$ & $0.9(0.4-1.7)$ & 0.78 & $2.1(1.1-4.2)$ & 0.03 \\
Tumour location (right vs. left) & $1.5(0.6-3.7)$ & 0.43 & $0.9(0.4-2.3)$ & 0.86 \\
Metastatic at diagnosis (yes vs. no) & $2.7(1.3-5.9)$ & 0.009 & $2(1-4)$ & 0.05 \\
RAS status (wildtype vs. mutant) & $0.7(0.3-1.4)$ & 0.31 & $1.7(0.8-3.4)$ & 0.14 \\
Lung only metastasis (yes vs. no) & $0.8(0.2-3.1)$ & 0.81 & $0.7(0.2-2.6)$ & 0.64 \\
\hline
\end{tabular}

PFS (progression-free survival), OS (overall survival), HR (hazard ratio).

The reason for Lonsurf discontinuation was progression of disease in $33.3 \%$, general decline in $28.6 \%$, and death within 30 days in $7.1 \%$. Median time from treatment discontinuation to death was two months (range 1-13).

\section{Discussion}

Since the approval of Lonsurf by NICE under National Cancer Drugs Fund (CDF) in 2016, it has become the drug of choice by many as the third line treatment for metastatic CRC. Previous real-world data on Lonsurf have been published, but to our knowledge this is the first published series with a minimum of two years of follow-up data, having followed up every patient until death.

While the median age at diagnosis was 61, the age at which Lonsurf was commenced was 66. The CDF criteria requires a patient to be of PFS 0 or 1 to be eligible for Lonsurf. In this review, twelve patients had PS greater than 1 immediately prior to starting cycle 1. As our cohort includes heavily pre-treated patients with advanced disease, PS may be borderline and prone to fluctuation. There is a 2-3-week delay between chemotherapy referral and the start of treatment, during which patient fitness can further decline. This finding has allowed us to review our cycle 1 pre-assessment protocol in order to flag up changes in PS to the clinical team.

The median PFS for patients with PS greater than 1 prior to cycle one was 3.0 months (range 1-7.8) compared to 3.2 months in the overall study population. Median OS in this subgroup was 3.0 months (range 1-12), compared to 5.8 months (1-28) in the overall group. Patients with deteriorating PS beyond 1 do not appear to benefit significantly from Lonsurf. These results support the recommendation that patients with PS above 1 should not be offered Lonsurf and should be offered best supportive care alone.

Our data is comparable to the results from other investigators summarised in Table 1. The results reported here showed a slightly better median PFS of 3.2 months compared to the 2 months reported in the RECOURSE trial. However, we reported an inferior median OS to that of the RECOURSE trial, namely 5.8 months compared to 7.1 months respectively. The RECOURSE trial was an international study which included patients from across the world, including Japan, USA and the EU and is only one of two prospective studies investigating the benefit of Lonsurf. Though our reported data concern a smaller cohort, their strength lies in the long-term real-world outcomes reported.

Interestingly, seven patients (12.5\%) in our series had a good response to Lonsurf, with PFS > 9 months and OS of 13.5 months (range 10-27). We looked for any factors that correlated with better treatment response. Multivariate analysis did not identify factors that were statistically significant apart from those presenting with metastatic disease and aged above 65 at diagnosis. This was however likely due to small numbers underpowering the statistical analysis. Several observations were nonetheless made which may be helpful to be further validated in a larger cohort. For example, all seven patients above had left-sided disease, with four patients having metastatic disease at the time of diagnosis, and only one patient with a RAS mutation. Left-sided tumours are typically less immunogenic and therefore may explain the better response to conventional chemotherapy such as Lonsurf. There is no apparent correlation between sites of metastases and prognosis. However, in this study, three patients had lung-only metastases, and appeared to have a good response 
to Lonsurf, with median PFS of five months (range 5-9), versus 3.2 months seen in the overall group. Median OS was 12 months (range 9-18) in this lung metastases-only subgroup, versus 5.8 months overall. The numbers are small, and this finding that patients with lung-only metastases may have a better treatment response than those with other sites of metastases has not thus far been reported. One possible explanation for better OS in this subgroup is lower burden of metastatic disease. Lower tumour burden $(<3$ metastatic sites at trial randomisation) has been reported by Tabernero et al. in their post-hoc exploratory analysis of the RECOURSE trial to be a good prognostic characteristic (GPC) [16]. Patients with lung metastases also appeared to have better PFS and OS response to Lonsurf, and those with lower tumour burden had more favourable response [16]. It is interesting to note that the definition of good prognostic characteristics in this post-hoc analysis also included indolent disease ( $\geq 18$ months from diagnosis of metastatic disease to trial randomisation) and did not look at lung-only metastases. Nevertheless, these are important observations that may help with selecting patients that would most benefit from Lonsurf.

After treatment discontinuation, the patients studied had a short time to death overall. There was however a subgroup that were able to receive further systemic treatment, and this subgroup had a good median OS of 12 months (range 1-17). There is no clear factor identified associated with a good response to further treatment. In addition, it is difficult to ascertain whether the additional OS gained is due to further treatment received, patient selection, or tumour biology. It would be interesting to look at whether this subgroup shares the same characteristics of the GPC subgroup discussed above.

This is the largest published real-world data from a single institution in the UK with a minimum of two-year follow-up. While Lonsurf offers a small PFS and OS benefit overall, we identified a cohort of patients who experienced a long period of disease control. All of these patients had left-sided disease, and most were RAS wild-type. In addition, a number of patients did go on to receive further systemic therapy after discontinuing Lonsurf, with good effect. It is not clear currently what baseline demographics or tumour characteristics confer this advantage, but this may be due to self-selection of patients with more indolent disease and better fitness.

There is growing evidence, both in recent publications and ongoing trials that may expand the role of Lonsurf in metastatic colorectal cancer. In the third-line setting, Pfeiffer et al. have demonstrated a two-month improvement of median PFS (2.6 vs. 4.6 months) by adding Bevacizumab to Lonsurf in the third-line setting [17]. While this is a Phase II study, the results of an ongoing Phase III study are eagerly anticipated. Similar efficacy was reported by Yoshida et al. in their single-arm Phase II study [18]. While the current role of Lonsurf is as monotherapy, this may change if studies are able to demonstrate increased efficacy when combined with other agents such as Bevacizumab. Importantly, the improvement in OS will be a key consideration by funding agencies. Its role in earlier lines of treatment remains to be answered by ongoing trials [19-21]. Our study suggests some tumour-factors that may help select patients who would most benefit from Lonsurf. For instance, with our observation that left-sided tumours demonstrated a better response to Lonsurf, it would be interesting to compare the response between left and right-sided tumours with the addition of Bevacizumab. The ongoing Phase III study may help further clarify the question of patient selection.

\section{Conclusions}

In summary, Lonsurf is an option for patients progressing beyond second line treatment. In this study, we found that patients with left-sided disease and RAS wild-type had a longer PFS. Although the numbers are small, patients with lung-only metastasis had better PFS and OS. Multivariate analysis was underpowered to confirm these findings and these factors would need to be further validated in a larger cohort. After progression, 12.5\% received further fluoropyrimidine-based treatment, suggesting that this is an option for selected patients. Patient selection is clearly key to identifying those who will best benefit from this third-line treatment. 
Supplementary Materials: The following are available online at https:/ / www.mdpi.com/article/10 .3390 / curroncol28030208/s1, Table S1. Details of Lonsurf treatment.

Author Contributions: Conceptualization, D.T. and S.E.; methodology, D.T, J.M. and S.E.; investigation, D.T. and L.W.; data curation, D.T., J.M. and L.W.; formal analysis, J.M.; writing-original draft preparation, D.T. and L.W.; writing-review and editing, D.T., L.W. and S.E.; supervision, S.E. All authors have read and agreed to the published version of the manuscript.

Funding: This research received no external funding.

Institutional Review Board Statement: Ethical review and approval were waived due to retrospective nature of the study.

Informed Consent Statement: Patient consent was waived due to due to retrospective nature of the study.

Data Availability Statement: The data presented in this study are available on request from the corresponding author. The data are not publicly available due to privacy and ethical reasons.

Acknowledgments: We gratefully acknowledge Hannah Knight and Susan Taylor for their help in identifying patients suitable for this study, and Simon Skene and Joanna Harris for identifying statistics input for this publication.

Conflicts of Interest: The authors declare no conflict of interest.

\section{References}

1. Cancer Research UK. Cancer Mortality for Common Cancers. Available online: https://www.cancerresearchuk.org/healthprofessional/cancer-statistics/mortality/common-cancers-compared/ (accessed on 31 July 2020).

2. Grothey, A.; Sargent, D.; Goldberg, R.M.; Schmoll, H.J. Survival of patients with advanced colorectal cancer improves with the availability of fluorouracil-leucovorin, irinotecan, and oxaliplatin in the course of treatment. J. Clin. Oncol. 2004, 22, 1209-1214. [CrossRef] [PubMed]

3. Van Cutsem, E.; Cervantes, A.; Nordlinger, B.; Arnold, D. Metastatic colorectal cancer: ESMO Clinical Practice Guidelines for diagnosis, treatment and follow-up. Ann. Oncol. 2014, 25, iii1-iii9. [CrossRef] [PubMed]

4. Temmink, O.H.; Emura, T.; de Bruin, M.; Fukushima, M.; Peters, G.J. Therapeutic potential of the dual-targeted TAS-102 formulation in the treatment of gastrointestinal malignancies. Cancer Sci. 2007, 98, 779-789. [CrossRef] [PubMed]

5. Yoshino, T.; Mizunuma, N.; Yamazaki, K.; Nishina, T.; Komatsu, Y.; Baba, H.; Tsuji, A.; Yamaguchi, K.; Muro, K.; Sugimoto, N.; et al. TAS-102 monotherapy for pretreated metastatic colorectal cancer: A double-blind, randomised, placebo-controlled phase 2 trial. Lancet Oncol. 2012, 13, 993-1001. [CrossRef]

6. Mayer, R.J.; Van Cutsem, E.; Falcone, A.; Yoshino, T.; Garcia-Carbonero, R.; Mizunuma, N.; Yamazaki, K.; Shimada, Y.; Tabernero, J.; Komatsu, Y.; et al. Randomized trial of TAS-102 for refractory metastatic colorectal cancer. N. Engl. J. Med. 2015, 372, $1909-1919$. [CrossRef] [PubMed]

7. New Zealand: Drugsite Trust. FDA Approves Lonsurf. Available online: https://www.drugs.com/newdrugs/fda-approveslonsurf-tipiracil-trifluridine-advanced-colorectal-cancer-4265.html/ (accessed on 31 July 2020).

8. UK: National Institute for Health and Care Excellence. Trifluridine-Tipiracil for Previously Treated Metastatic Colorectal Cancer. Available online: https:/ / www.nice.org.uk/guidance/TA405/chapter/1-Recommendations (accessed on 31 July 2020).

9. Bachet, J.B.; Wyrwicz, L.; Price, T.; Cremolini, C.; Phelip, J.M.; Portales, F.; Ozet, A.; Cicin, I.; Atlan, D.; Becquart, M.; et al. Safety, efficacy and patient-reported outcomes with trifluridine/tipiracil in pretreated metastatic colorectal cancer: Results of the PRECONNECT study. ESMO Open 2020, 5, e000698. [CrossRef] [PubMed]

10. Stavraka, C.; Pouptsis, A.; Synowiec, A.; Aggelis, V.; Satterthwaite, L.; Khan, S.; Chauhan, M.; Holden, C.E.; Young, S.; Karampera, C.; et al. Trifluridine/tipiracil in metastatic colorectal cancer: An updated multicentre real-world analysis on efficacy, safety and predictive factors. Ann. Oncol. 2019, 30, v198-v252. [CrossRef]

11. Tilby, M.; Escola, C.; Ellison, C.; Narramneni, L.; King, M.; Grumett, S.; Jain, A. Trifluridine-tipiracil for the treatment of metastatic colorectal cancer patients: UK multicentre real-world experience. Ann. Oncol. 2019, 30, IV27. [CrossRef]

12. Samawi, H.H.; Brezden-Masley, C.; Afzal, A.R.; Cheung, W.Y.; Dolley, A. Real-world use of trifluridine/tipiracil for patients with metastatic colorectal cancer in Canada. Curr. Oncol. 2019, 26, 319-329. [CrossRef] [PubMed]

13. Andersen, S.E.; Andersen, I.B.; Jensen, B.V.; Pfeiffer, P.; Ota, T.; Larsen, J.S. A systematic review of observational studies of trifluridine/tipiracil (TAS-102) for metastatic colorectal cancer. Acta Oncol. 2019, 58, 1149-1157. [CrossRef] [PubMed]

14. Wallander, M.; Rolander, B.; Åvall-Lundqvist, E.; Elander, N.O. Real world aspects of palliative trifluridine plus tiperacil (TAS-102) in refractory metastatic colorectal cancer. J. Gastrointest. Oncol. 2020, 11, 616-625. [CrossRef] [PubMed]

15. Cremolini, C.; Rossini, D.; Martinelli, E.; Pietrantonio, F.; Lonardi, S.; Noventa, S.; Tamburini, E.; Frassineti, G.L.; Mosconi, S.; Nichetti, F.; et al. Trifluridine/Tipiracil (TAS-102) in Refractory Metastatic Colorectal Cancer: A Multicenter Register in the Frame of the Italian Compassionate Use Program. Oncologist 2018, 23, 1178-1187. [CrossRef] [PubMed] 
16. Tabernero, J.; Argiles, G.; Sobrero, A.F.; Borg, C.; Ohtsu, A.; Mayer, R.J.; Vidot, L.; Moreno Vera, S.R.; Van Cutsem, E. Effect of trifluridine/tipiracil in patients treated in RECOURSE by prognostic factors at baseline: An exploratory analysis. ESMO Open. 2020, 5, e000752. [CrossRef] [PubMed]

17. Pfeiffer, P.; Yilmaz, M.; Möller, S.; Zitnjak, D.; Krogh, M.; Petersen, L.N.; Poulsen, L.Ø.; Winther, S.B.; Thomsen, K.G.; Qvortrup, C. TAS-102 with or without bevacizumab in patients with chemorefractory metastatic colorectal cancer: An investigator-initiated, open-label, randomised, phase 2 trial. Lancet Oncol. 2020, 21, 412-420. [CrossRef]

18. Yoshida, Y.; Yamada, T.; Kamiyama, H.; Kosugi, C.; Ishibashi, K.; Yoshida, H.; Ishida, H.; Yamaguchi, S.; Kuramochi, H.; Fukazawa, A.; et al. Combination of TAS-102 and bevacizumab as third-line treatment for metastatic colorectal cancer: TAS-CC3 study. Int. J. Clin. Oncol. 2021, 26, 111-117. [CrossRef] [PubMed]

19. Yoshino, T.; Oki, E.; Nozawa, H.; Eguchi-Nakajima, T.; Taniguchi, H.; Morita, S.; Takenaka, N.; Ozawa, D.; Shirao, K. Rationale and design of the TRUSTY study: A randomized, multicentre, open-label phase II/III study of tri- fluridine/tipiracil plus bevacizumab versus irinotecan fluoropyrimidine plus bevacizumab as second-line treatment in patients with metastatic colorectal cancer progressive during or following first-line oxapliplatin- based chemotherapy. ESMO Open 2018, 3, e0000411. [CrossRef]

20. Van Cutsem, E.; Danielewicz, I.; Saunders, M.P.; Pfeiffer, P.; Argilés, G.; Borg, C.; Glynne-Jones, R.; Punt, C.J.A.; Van de Wouw, A.J.; Fedyanin, M.; et al. Trifluridine/tipiracil plus bevacizumab in patients with untreated metastatic colorectal cancer ineligible for intensive therapy: The randomized TASCO1 study. Ann. Oncol. 2020, 31, 1160-1168. [CrossRef] [PubMed]

21. André, T.; Saunders, M.; Kanehisa, A.; Gandossi, E.; Fougeray, R.; Amellal, N.C.; Falcone, A. First-line trifluridine/tipiracil plus bevacizumab for unresectable metastatic colorectal cancer: SOLSTICE study design. Future Oncol. 2020, 16, 21-29. [CrossRef] [PubMed] 